\title{
Dynamic Perturbation of the Electrical Double Layer with an Electrochemical AFM for Confined Metal Electrodeposition
}

Esther Alarcon, mark aarts

Submitted date: 09/06/2019 - Posted date: 11/06/2019

Licence: CC BY 4.0

Citation information: Alarcon, Esther; aarts, mark (2019): Dynamic Perturbation of the Electrical Double Layer with an Electrochemical AFM for Confined Metal Electrodeposition. ChemRxiv. Preprint.

We demonstrate the directed electrochemical deposition of copper nanostructures by using an oscillating nanoelectrode operated with an atomic force microscope (AFM). Strikingly, the writing is only possible in highly dilute electrolytes and for a particular combination of AFM and electrochemical parameters. We propose a mechanism based on cyclic charging and discharging of the electrical double layer (EDL). The extended screening length and slower charge dynamics in dilute electrolytes allows the nanoelectrode to operate inside, and disturb, the EDL even for large oscillation amplitudes ( $100 \mathrm{~nm})$. Our unique approach can not only be used for controlled additive nano-fabrication but also provides insights into ion behavior and EDL dynamics at the solid-liquid interface.

File list (2)

Aarts-ECAFM.pdf (1.95 MiB)

view on ChemRxiv • download file 


\title{
Dynamic perturbation of the electrical double layer with an electrochemical AFM for confined metal electrodeposition
}

\author{
Mark Aarts ${ }^{1}$ and Esther Alarcon-Llado ${ }^{1}$ \\ ${ }^{1}$ Center for Nanophotonics, NWO-I Amolf, Science Park 104, 1098 XG Amsterdam, The \\ Netherlands
}

May 17, 2019

\begin{abstract}
Understanding and directing electrochemical reactions below the micrometer is a long-standing challenge in electrochemistry. Confining reactions to nanoscale areas paradoxically requires both isolation and communication with the bulk electrolyte in terms of potential and access of ions, respectively. Here, we demonstrate the directed electrochemical deposition of copper nanostructures by using an oscillating nanoelectrode operated with an atomic force microscope (AFM). Strikingly, the writing is only possible in highly dilute electrolytes and for a particular combination of AFM and electrochemical parameters. We propose a mechanism based on cyclic charging and discharging of the electrical double layer (EDL). The extended screening length and slower charge dynamics in dilute electrolytes allows the nanoelectrode to operate inside, and disturb, the EDL even for large oscillation amplitudes ( $100 \mathrm{~nm}$ ). Our unique approach can not only be used for controlled additive nano-fabrication but also provides insights into ion behavior and EDL dynamics at the solid-liquid interface.
\end{abstract}

\section{Introduction}

Electrochemical deposition can revolutionize the fabrication of (opto)electronic nanodevices if carefully controlled at the nanoscale. Among its advantages are its relatively small infrastructure costs, low thermal budget and it offers fine dynamic control over reaction kinetics and thermodynamics, which does not exist with any other synthetic method. However, the biggest challenge in integrating electrodeposition and nanotechnology is the controlled down-scaling of chemical reactions down to the nanoscale. ${ }^{1,2}$ This control would provide additional freedom in designing free-form nano-architectures with extraordinary properties and functionalities. ${ }^{3,4,5,6,7}$

One approach to confine growth is by spatially restricting the electrochemical cell or the feeding of precursors, such as in meniscus confined electrodeposition, local dispensing of ions, or electrohydrodynamic jet printing. $8,9,10,11,12,13$ A different strategy is the direct electrochemical writing with small electrodes. Complex 3D structures have been successfully made at the scale of tens to few hundredths of microns by using scanning microelectrodes, where the size of the electrode and its distance to the sample define the fabrication resolution. ${ }^{14}$

At the nanoscale, scanning probes have demonstrated high precision in nanostructuring metal, semiconductor and oxide surfaces under gas or liquid conditions. ${ }^{15,16,17,18}$ based on a variety of tip-surface interactions. However, in most cases, growth is restricted to a few nanometers in height (such as clusters). 
The additive electrochemical nano-fabrication with scanning probes beyond cluster size has been proven to be difficult, opposite to what could be expected from conventional electrochemistry. The main reason being that when the tip/substrate gap is of the order of nanometers, the reaction dynamics are governed by a complex interplay of electrochemical potential distributions, ${ }^{19}$ poor communication with the bulk solution and mass transport limitations of ions into the small gap. ${ }^{20,21,22}$ In general, effects of the structure and dynamics of the electrical double layer (EDL) at the solid-liquid interface on nanoscale electrochemistry are not fully understood. 23,24 In the past years, several groups have focused on using scanning probe microscopy techniques to probe the solid-liquid interface $25,26,27,28,29,30,31,32$, in terms of specific adsorption ${ }^{33}$, charge density, and screening ${ }^{34,35}$. In all these works, care was taken to ensure minimal perturbation of the EDL by the probing tip. However, it has been suggested that in some cases, the probe may strongly perturb the charge distribution at the interface. ${ }^{36,37}$

Schuster and co-workers demonstrated the etching and deposition of metals with micrometer resolution by locally polarizing the EDL at the water-substrate interface upon the application of a voltage pulse at a microelectrode ${ }^{38}$. The authors described the reaction confinement by considering a simple RC equivalent circuit, concluding that nanometer resolution would require fs pulses. However, this timescale is much faster than the typical electric double layer dynamics $\left({ }^{\sim} n s-\mu s\right)$. ${ }^{39}$ Interestingly, nanoscale local etching and depostition has been demonstrated with $\mu$ spulses to an STM tip. ${ }^{40,41,42}$

In this work, we take advantage of the capabilities of an electrochemical atomic force microscope (EC-AFM) tip to dynamically perturb the EDL in order to control growth at the nanoscale. We achieve this by using a highly dilute electrolyte and highly asymmetric tip-substrate system (in terms of material and potential). We demonstrate direct electrodeposition of copper on gold, with lateral dimensions down to tens of nanometers and aspect ratio above 0.5 . We show a critical dependence of the ability to confine growth on the salt concentration, as well as the dynamics of the tip movement during the writing. We propose a local growth mechanism facilitated by a combination of periodic disruption and build-up of the EDL, followed by forced electrodeposition due to the strong perturbation of the electrochemical potential between the tip and the sample.

The ability to control solution-based growth down to the nanoscale can revolutionize nanofabrication, by enabling the making of combined logical circuits, LEDs, photodetectors and many other (opto)electronic devices on the same platform with nanoscale resolution. Additionally, local deposition with dynamic EC-AFM can be used as an indirect means to probe EDL dynamics in general, which has a strong impact in any electrochemical process such as in batteries, or elecrocatalysis.

\section{Results}

Direct writing of copper nanostructures We control the local growth of copper on gold by applying a potential difference directly between a Au substrate and an AFM tip in a dilute aqueous electrolyte $(1 \mu \mathrm{M}$ $\mathrm{CuSO}_{4}$ ). The tip apex, which is made of $\mathrm{Pt}$, is the only part of the cantilever that is exposed to the liquid. ${ }^{43}$ During writing, the AFM is operated in peakforce (PF) tapping mode, where the cantilever is driven by a sinusoidal signal far below its resonance frequency ( $2 \mathrm{kHz}$ in this work) (Fig. 1a, and methods). In this mode, the force exerted by the tip is used as the feedback signal ( 3 to $30 \mathrm{nN}$ in this work), while the intermittent contact results in minimal lateral forces on the substrate. By moving the tip along a programmed $x y$ trajectory, arbitrary shapes can be written ( Figs. 1b and 1c). 
Single lines down to a full width half maximum (FWHM) of $\sim 50 \mathrm{~nm}$ and as close as $125 \mathrm{~nm}$ are obtained with aspect ratios above 0.5 (line profiles in Figs. $1 \mathrm{~b}$ and $1 \mathrm{c}$ ). It should be noted that the extent of the deposition profile is in the order of the expected tip radius, which is typically regarded as the lower limit on resolution in most SPM-based nanofabrication ${ }^{44,18}$. The clear Z-contrast of the deposited structure with the gold substrate as obtained from backscattered electrons in a scanning electron microscope (SEM) highlights the chemical nature of the deposited copper wire, as is also found by energy dispersive $\mathrm{X}$-ray analysis from a larger structure (supplementary information SI-1).

a)

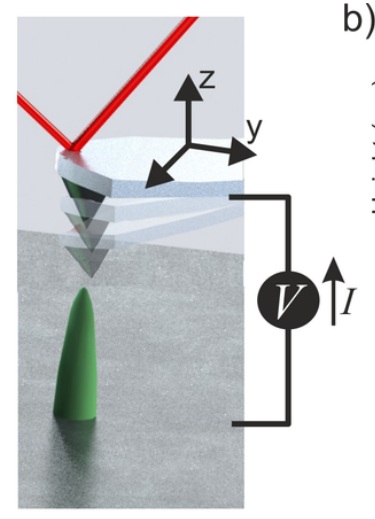

c)

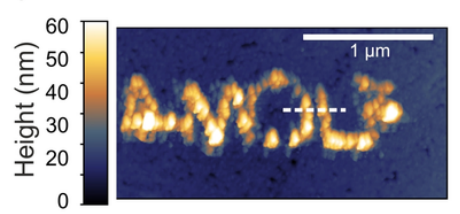

b)
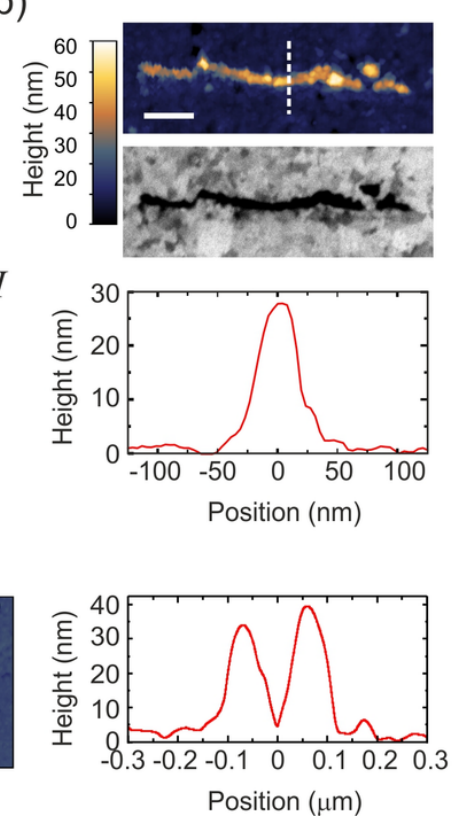

d)

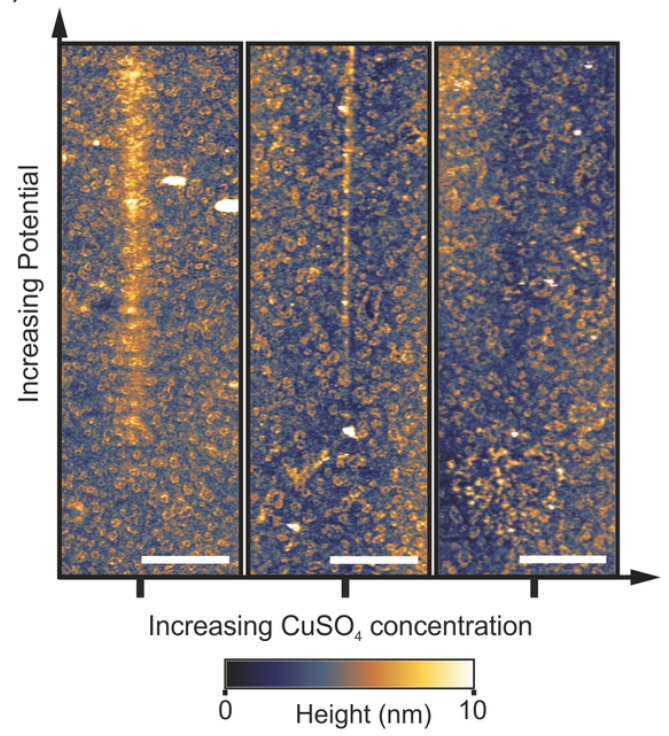

Figure 1: Direct writing of copper with dynamic EC-AFM a) Cartoon representing the EC-AFM writing set-up b) Ex-situ AFM-topography, cross-section height profile, and in-column SEM, of a $1 \mu \mathrm{m}$ long line deposited in a single EC-AFM pass at $3 \mathrm{~nm} / \mathrm{s}$ with an applied potential of $1.7 \mathrm{~V}$. c) Ex-situ AFM-topography and cross-section height profile of the written AMOLF logo, deposited in a consecutive double pass at 30 $\mathrm{nm} / \mathrm{s}$ and $3 \mathrm{~nm} / \mathrm{s}$ with an applied potential of $1.7 \mathrm{~V}$. The writing in b) and c) were done in $1 \mu M \mathrm{CuSO}_{4}$ solutions scale bars $200 \mathrm{~nm}$ d) In-situ AFM topography, after following a $12 \mu \mathrm{m}$-long path accross the center of each image while ramping the potential from 1 to $2.5 \mathrm{~V}$ (in the first two panels) and 1.2 to $2.7 \mathrm{~V}$ (in the rightmost panel). Three different concentrations of $\mathrm{CuSO}_{4}$ were used (from left to right, 1,4 and 12 $\mu \mathrm{M})$. The deposition disappears by increasing the concentration. The scale bar is $2 \mu \mathrm{m}$.

To enable the local writing however, we find two counter-intuitive results that disagree with previously reported micro-, and nano- SPM based electrochemical deposition mechanisms. First, we find that the local deposition only occurs when reducing the salt concentration down to a few $\mu \mathrm{M}$. The panels in Fig. $1 \mathrm{~d}$ show the topography after the tip is translated over a $12 \mu \mathrm{m}$ long path with the PF feedback enabled and ramping the voltage ( $1<\mathrm{V}<2.7 \mathrm{~V}$ ) along the path. The figure clearly shows a topography emerging with increasing potential in the case with lowest ion concentration, which gradually disappears by increasing concentration. In fact, at concentrations above $10 \mu \mathrm{M}$, we are unable to observe any growth within our typical AFM scan range $(\sim 15 \mu \mathrm{m})$ and parameters (supplementary information $\mathrm{SI}-2$ ). While increasing the concentration of $\mathrm{CuSO}_{4}$ should accelerate growth, mainly through facilitating mass transport, it is not in line with our observations. This is therefore a critical result, and rules out other known writing mechanisms, such as AFM-induced local depassivation of a metal substrate ${ }^{16,15}$ or electric-field enhanced mass transport $45,46,47$. 
Second, no deposition is observed when the tip is kept at a fixed tip-substrate distance (ranging from 3-300 $\mathrm{nm}$ ). In fact, we will illustrate that the AFM tip dynamics play a critical role in the direct writing mechanism.

Influence of electrochemical parameters First, we investigate the electrochemical nature of the deposition process. The electrochemical behavior of the tip-substrate system is shown in Fig $2 a$, where no current flows until reaching a minimum potential of around $1.2 \mathrm{~V}$, after which the current increases exponentially. This is consistent with a minimum thermodynamic potential difference between the oxidation of water at the tip and copper reduction at the substrate.

From ex-situ AFM topogrpahy we determine the deposited volume as a function of the total charge passed through the system, which is controlled by varying either the voltage ( 1.3 to $2.1 \mathrm{~V}$ in steps of $0.1 \mathrm{~V}$, Fig. 2b) or writing time by means of the translation speed $(3,10,30$, and $100 \mathrm{~nm} / \mathrm{s}$, Fig. 2c). The linear dependence of the volume on charge supports the electrochemical nature of the process. However, the amount of charge required to obtain an equivalent volume of solid copper from $\mathrm{Cu}^{2+}$ is five orders of magnitude smaller than that derived from the measured current. This suggests that large stray currents are present across the substrate that do not result in copper growth, which is not surprising given the large surface area of our substrate electrode $\left({ }^{\sim} 5 \mathrm{~cm}^{2}\right)$. Moreover, Fig. $2 \mathrm{~b}$ shows that the deposition is suppressed at high currents/potentials most likely due to the competing proton reduction reaction, which leads to a "working" potential window. Actually, the formation of bubbles is observed underneath the tip at higher current densities (typically 20 $\mathrm{nA})$.

a)

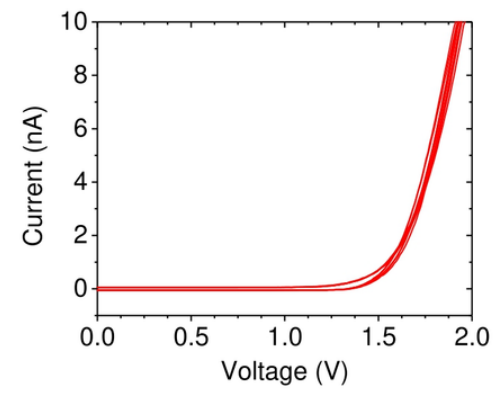

b)

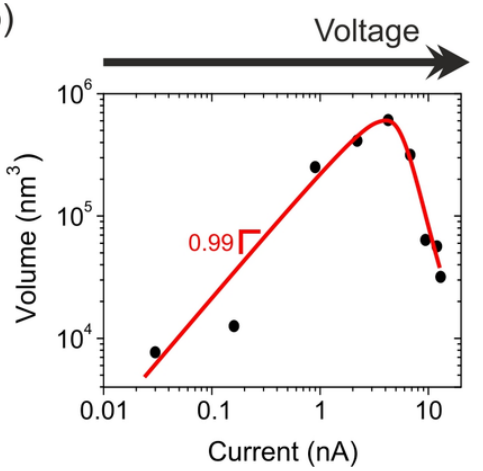

c)

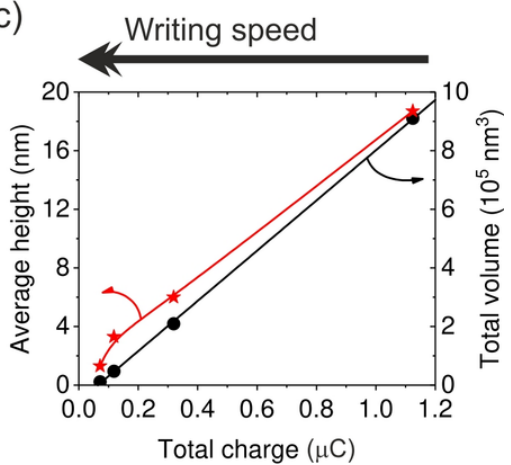

Figure 2: Electrochemical nature of the local $\mathbf{C u}$ deposition a) Current-voltage characteristics of the tip-Au substrate two electrode system. At positive bias, current in the electrolyte flows from the tip to the substrate b) Linear scaling of the volume as a function of current in $1000 \mathrm{~nm}$-long lines for a fixed set of AFM parameters. Different currents are obtained by increasing the applied potential. At high potentials/currents the deposition efficiency drops most likely due to the competing hydrogen evolution reaction. The volume is obtained from ex-situ AFM topography. c) Volume and height of $1000 \mathrm{~nm}$-long lines as a function of the total charge transferred for a given voltage and same peakforce parameters as in $b$ ). The different points are obtained by different writing speeds, from 3 to $100 \mathrm{~nm} / \mathrm{s}$.

The additive nature of the fabrication is highlighted by the increase in volume with time when driving the system at a fixed potential. In particular, the volume increases linearly with charge but only after a certain threshold (Fig. 2b, black curve). We attribute the presence of a minimum amount of charge required for growth to kinetic limitations of the nucleation. By contrast, the average height of the deposits (red curve) rises fast right after the threshold followed by a linear increase as a function of total charge. This dependence is indicative of a switch between the isotropic growth characteristic of small clusters (height $\propto$ Volume $e^{\frac{1}{3}}$ ) to vertical growth (height $\propto$ Volume). 
Influence of tapping dynamics In the following, we investigate the impact of AFM tapping parameters on local growth to better understand the mechanism of confined electrochemistry. We adjust the tip position dynamics through the peakforce amplitude (PFA) and the force setpoint (FS) parameters (Fig. 3a). The time-averaged tip-sample distance or gap size $(<z>)$ and time in contact $(C T)$ are estimated from the PFA and FS settings as described in the supplementary file SI-3. The effect of the FS on $\langle z\rangle$ is quite significant. For instance, at the nominal spring constant of $1.5 \mathrm{~N} / \mathrm{m}$ of our tips, the maximum gap size is reduced by 20 $\mathrm{nm}$ when the $\mathrm{FS}$ is increased by $30 \mathrm{nN}$, a value that may represent a large fraction of the oscillation amplitude (see supplementary file SI-3).

a)

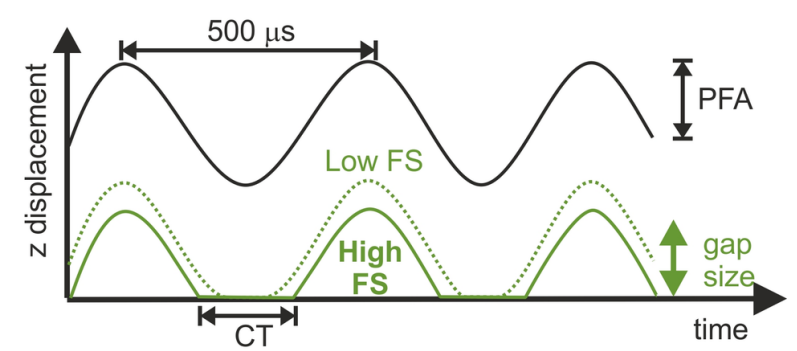

b)
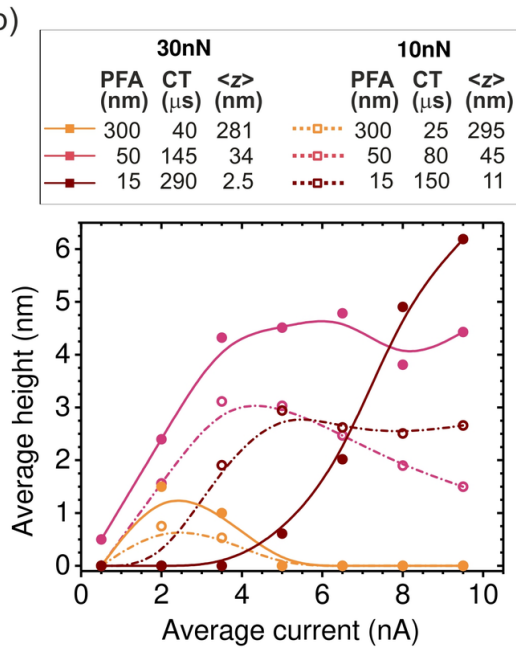

c)

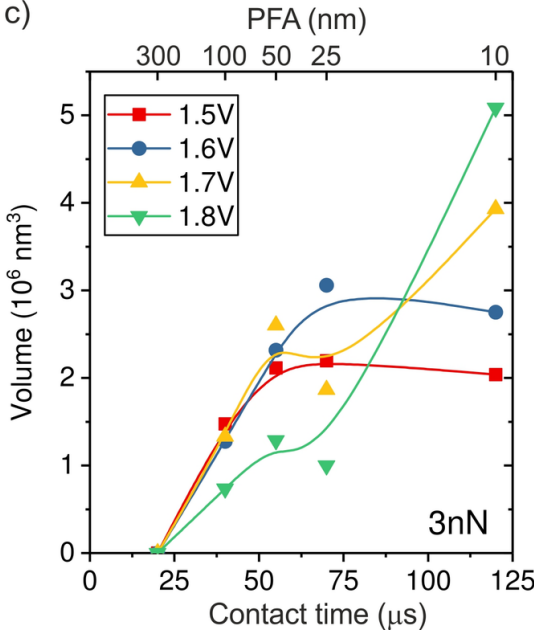

Figure 3: Influence of the AFM tip dynamics on the local deposition a) Representation of the tip dynamics over a few tapping cycles. The peakforce oscillation amplitude (PFA) sets the amplitude of the drive signal (black curve). While the cantilever follows the drive signal, the tip-substrate distance (green curves) and contact time (CT) depends as well on the force setpoint (FS). As a result, the center of oscillation moves up and down as a function of both parameters. b) Height of $\mathrm{Cu}$ lines, averaged along a $1000 \mathrm{~nm}$ path written at $10 \mathrm{~nm} / \mathrm{s}$, as a function of applied current for different PFA and FS. The estimated CT and average gap size $(<z>)$ during the oscillation are listed above. Both the height of the deposit and the optimal current change as a function of the oscillation parameters $\mathbf{c}$ ) Total deposited volume in $\mathrm{Cu}$ lines written along a $500 \mathrm{~nm}$ path as a function of CT, which is controlled by changing the PFA from 10 to $300 \mathrm{~nm}$. All lines are written with the same $\mathrm{FS}$ of $3 \mathrm{nN}$, a translation speed of $10 \mathrm{~nm} / \mathrm{s}$ and at fixed bias voltages from 1.5 to $1.8 \mathrm{~V}$ (average currents of $\sim 1,2.5,4,6.5 \pm 0.5 \mathrm{nA})$. The figure displays a threshold contact time for deposition of $\sim 25 \mu \mathrm{s}$

The influence of the tip dynamics on the local growth is presented by plotting the average maximum height of a line deposited along a $1000 \mathrm{~nm}$-long path as a function of current (Fig. 3b), as we do not observe a consistent trend in the width of the deposit as a function of the oscillation parameters or the current (supplementary file SI-4). Here, the paths are followed at either 10 or $30 \mathrm{nN}$, corresponding to dashed and thick lines, respectively, and with three different oscillation amplitudes $(15,50$ and $300 \mathrm{~nm})$. The corresponding average 
gap size and contact time is listed in the table of Fig. 3b. Similar to Fig. 2a, local growth is favored within a limited electrochemical window, leading to an optimal drive condition. Surprisingly, the optimal drive strongly depends on the AFM operation parameters. At large oscillation amplitudes, where the tip is floating above the substrate most of the time, little deposition is seen regardless of force. This reinforces the notion that the process is not primarily driven by surface depassivation from simple mechanical tip-sample interaction. We find that the share of time spent in contact with the sample appears to be a key parameter for determining the optimal driving condition for local growth. This is particularly evident at $15 \mathrm{~nm}$ amplitude, where heightcurrent behavior for the two forces is quite different. Rather, the data at $10 \mathrm{nN}$ resembles that of $30 \mathrm{nN}$ with a similar contact time (i.e. amplitude of $50 \mathrm{~nm}$ ).

In order to further investigate the link between CT and local deposition, we plot the total volume as a function of $\mathrm{CT}$ for lines written at a fixed force of $3 \mathrm{nN}$ and various potentials (Fig. 3c). Different dependencies can be seen as a function of driving potential. At low drives ( 1.5 and $1.6 \mathrm{~V})$, the volume increases with CT, and saturates after $\sim 50 \mu s$. At high drives $(1.7$ and $1.8 \mathrm{~V})$, growth is hampered for a $\mathrm{CT} \sim 60 \mu s$, and rapidly increases at longer times. This is consistent with the data from Fig. 3b, where growth at long contact times (dark red solid data) only occurs at high currents. More striking in Fig. $3 c$ is the observation that growth is absent for all potentials when the contact time is shorter than $25 \mu \mathrm{s}$. Notably, this threshold value for the contact time is in line with the observation that no deposition is obtained with the tip held at fixed distance above the surface (limit of zero contact).

\section{Discussion}

While we have proven that the localized $\mathrm{Cu}$ deposition process studied here is electrochemically driven, we find a particularly intriguing critical dependence of deposition on the contact time. Namely, the longer the contact time the more material is deposited. This is rather counter-intuitive as we suspect the tip to be in electrical contact with the substrate during the time in contact (see supplementary file SI-5 for more details), and therefore suspend electrochemical growth.

In potential-pulsed electrochemistry, the "off-time" is considered as means of double layer relaxation and electrochemical equilibrium and has led to confined electrochemical machining with STM tips, control over crystallographic defect formation or atomic layer electrochemical deposition, among other phenomena. ${ }^{48,40} 49,50$

Upon application of an external voltage at an electrode, ions in solution are expected to re-order and form the electrical double layer, ie. a region containing a non-zero charge density that screens the electrode potential. The screening occurs in a diffuse layer extending from the electrode to the bulk solution (Fig. 4a). While this potential drop typically occurs within a distance of $1-2 \mathrm{~nm}$, it extends to $\sim 150 \mathrm{~nm}$ for the highly dilute $1 \mu \mathrm{MCuSO}_{4}$ solution. This is due to the screening length or Debye length, $\lambda_{D}$, being inversely proportional to the square root of the concentration ( $\lambda_{D} \sim \frac{9.7}{n \sqrt{C}}[\mathrm{~nm}]$ for aqueous solutions with $\epsilon=80$ at room temperature, where $n$ is the ion valence and $C$ the concentration in units of $\mathrm{mM}) .{ }^{51,52}$

The disassembly of the double layer is associated with the diffusion of ions from the interface to the bulk through the diffuse layer over a time $\tau_{D}=\frac{\lambda_{D}^{2}}{D}$, with $D$ being the diffusion coefficient. ${ }^{53}$ Given the extended screening length in the dilute electrolyte, $\tau_{D}$ is $22 \mu \mathrm{s}(33 \mu \mathrm{s})$ for the $\mathrm{SO}_{4}^{2-}\left(\mathrm{Cu}^{2+}\right)$ ions (using $\mathrm{D}_{\mathrm{SO}_{4}^{2-}}=$ $\left.1.065 \cdot \frac{10^{-5} \mathrm{~cm}^{2}}{\mathrm{~s}}, D_{C u^{2+}}=0.72 \cdot \frac{10^{-5} \mathrm{~cm}^{2}}{\mathrm{~s}}\right)$, which is remarkably close to the contact time threshold for deposition found in Fig. 3c.

Additionally, considering oscillation amplitudes ranging from 10 to $300 \mathrm{~nm}$, it becomes apparent that the typical electrode separation lies within the screening length of the electrolyte. In fact, due to incomplete 
a)

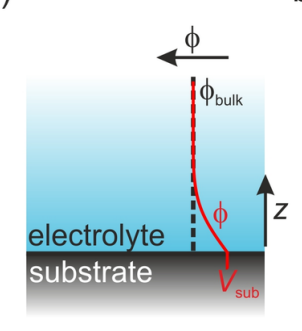

b)

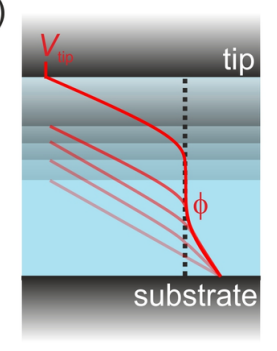

c)

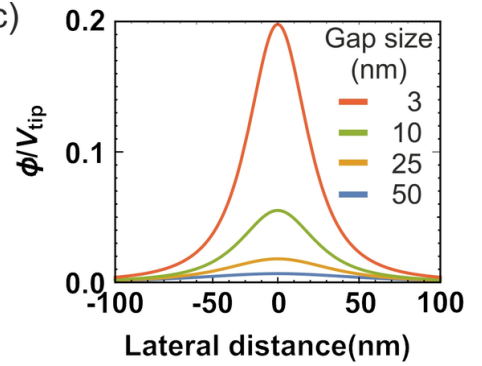

Figure 4: Tip-enabled local deposition mechanism in dilute electrolytes. Schematic representation of the potential drop at the solid-liquid interface for the general case of a metal electrode (a) and within the tip-substrate gap (b). When the gap size is smaller than twice the Debye screening length, the potential linearly decays with the distance. c) Calculated potential profiles at $0.6 \mathrm{~nm}$ above the substrate for various gap sizes $(3,10,25$ and $50 \mathrm{~nm})$ as obtained from the 2D Laplace's equation. We have considered a tip of $25 \mathrm{~nm}$ in radius held at a potential of $V_{t i p}$ and a grounded substrate. The potential is normalized to the tip bias.

screening the electrostatic attractive force is large enough to be sensed by the AFM. We observe this as an attractive force offset of $\sim 4 \mathrm{nN}$ in the baseline when executing a single force curve on the substrate and toggling the applied potential at contact (supplementary SI-6). In the case where electrode separation is $\leq 2 \lambda_{D}$ (also been referred as double layer cross-talk), the electrochemical conditions in the gap are not well-defined and may lead to unconventional electrochemistry. The double layer overlap between an STM tip and metal substrate has been suggested to cause an enhanced local dissolution of the substrate while being held at a known potential, where no dissolution is expected. ${ }^{36,54,37}$ Particularly different in these works with respect to ours, is the much smaller gap sizes ( $<2 \mathrm{~nm}$ to ensure feedback from tunneling current) and the unlimited access to the solid metal atoms for the oxidation reaction.

The interesting implication of incomplete screening in the gap facilitated by the continuous disruption of the EDL, is that the tip and substrate effectively form a capacitor. In other words, the ions inside the gap are subject to a (linear) potential profile between the electrodes purely defined by gap size and bias voltage (Fig. 4b). In the limiting case of no free charges in the gap, the potential can be calculated by solving Laplace's equation. Considering the plane of closest approach for a solvated ion $(0.6 \mathrm{~nm}$ arising from the size of the water molecule ${ }^{52}$ ), the solution potential at the interface is raised by $\sim 5$ of the tip voltage at a 10 $\mathrm{nm}$ gap size, and up to $20 \%$ at $3 \mathrm{~nm}$, as illustrated in Fig. $4 \mathrm{~d}$. Since we typically use bias voltages up to $\sim 2 \mathrm{~V}$, the unscreened tip perturbation can potentially increase the electrochemical potential of ions inside the gap by up to a a few hundred $\mathrm{mV}$ through direct electrostatic interaction. This effect greatly enhances the local charge transfer, as Faradaic currents exponentially increase with potential, but it quickly disappears as the tip moves away from the substrate.

Conversely, despite the reaction rate is accelerated as the tip approaches the substrate, we suspect that access of ions into the gap becomes restricted for gap sizes smaller than $10 \mathrm{~nm}$ (see approach curves in supplementary file SI-5). As a result, at small oscillation amplitudes higher drives may be necessary to facilitate mass transport and result into noticeable deposition, which is consistent with our observations from Fig.3.

Finally, we comment on the fact that higher concentrations hamper the local deposition within our available parameter window. Considering the discussion above, it is noteworthy to mention that at just slightly higher concentrations, $10 \mu M$, the improved screening power of the electrolyte results in the Debye screening length 
being about three times shorter $\left(\lambda_{D} \sim 50 \mathrm{~nm}\right)$. Double layer cross-talk and direct electrostatic interaction in less dilute electrolytes is therefore only possible at shorter oscillation amplitudes in which mass transport limitations are restricting growth at the same time.

\section{Conclusions}

We have demonstrated the confined high-resolution direct additive writing of copper nanostructures by using a biased AFM tip in a dilute electrolyte, where the tip is in intermittent contact with the substrate through the oscillation of the cantilever. We find a particularly intriguing critical dependence of deposition on the tapping dynamics of the AFM and ion concentration. We propose a confinement mechanism that balances access of ions and shaping of the electrochemical potential in the tip-substrate gap. While this method is expected to be general for the electrochemical deposition of a variety of materials, opening up new routes for nanofabrication, probing local reactions through topography provides a way to understand the local dynamics and charge transfer at the electrical double layer, such as the Debye time.

\section{Methods}

Sample and electrolyte preparation The substrate consists of a Au film ( $\sim 50 \mathrm{~nm}$ thick) with a $\sim 5 \mathrm{~nm} \mathrm{Cr}$ adhesion layer deposited by thermal evaporation on top of an $\mathrm{n}$-type Silicon sample (Siegert Wafer) as a flat support. The solutions were prepared using $\mathrm{CuSO}_{4} * 5 \mathrm{H}_{2} \mathrm{O}$ powder (99.995\%, from Sigma-Aldrich) in MilliQ (TM) $(18.2 \mathrm{M} \Omega \mathrm{cm})$ water. The low concentration electrolyte was prepared before every experiment from a $1 \mathrm{mM} \mathrm{CuSO}_{4}(\mathrm{aq})$ stockpile and diluted (100-1000x) using fresh MilliQ water. Before preparing the dilute electrolyte, all containers were rinsed (5-10 x), ultrasonicated, and rinsed again (5-10 $x$ ) using fresh MilliQ water. Both the container and electrochemical cell only contained the dilute copper electrolytes, after initially cleaning them by an overnight soak in $1 \mathrm{M} \mathrm{HCl}$. All experiments were carried out in ambient conditions inside a fume hood. Samples and the electrochemical cell were stored in a $1 \%$ humidity nitrogen environment.

Electrochemical Cell Our electrochemical system consists of an AFM nanoelectrode tip immersed in a filled electrochemical cell and connected to an external potentiostat, which controls and monitors potential and current. At positive potentials (tip vs. substrate), we expect the oxygen evolution reaction to occur at the tip and either copper reduction or hydrogen evolution at the sample. The electrochemical cell was homemade, and consisted of Teflon (R), made leak tight by an O-ring. Top-contacts were made by either attaching a wire connection directly to the sample edge, or by using a spring-loaded pin outside of the O-ring.

AFM The measurements and writing were performed using a Bruker Dimension Icon AFM, controlled through the Nanoscope software. The nanoelectrode AFM probes are obtained from Bruker (peakforce SECM) and are electrically insulated except for the platinum coated apex, which has a nominal radius of $50 \mathrm{~nm}{ }^{43}$. The AFM spring constant is obtained for each cantilever i) directly in the electrolyte, by doing a thermal tune at a distance of $\sim 1 \mathrm{~mm}$ from the substrate after obtaining the deflection sensitivity on the gold substrate inside the electrolyte, or ii) before filling the cell using the thermal tune method in air at a distance of $\sim 1 \mathrm{~mm}$ from the substrate, after obtaining the deflection sensitivity on the gold substrate. The deflection sensitivity of the cantilever is then re-calibrated on the gold substrate, inside the electrolyte. The measured $f_{0}, Q$, and used $k$ are reported in Supplementary file SI-7 as proposed in reference ${ }^{55}$. Ex-situ topography and data in figures $1 \mathrm{~b}, 1 \mathrm{c}$, and 2 are obtained in air using scanasyst-air tips (Bruker, nominal tip radius $2 \mathrm{~nm}$ ). In-situ images and data in figures $1 \mathrm{~d}$ and 3 are collected using the SECM tip, directly after deposition. 
Potentiostat Potential/current control and readout were done with a $\mathrm{CH} 760 \mathrm{E}$ potentiostat. In all experiments the system was connected in a 2-electrode fashion, by shorting the reference and counter electrode.

Writing protocol For writing, the tip follows a programmed trajectory (NanoScript) in the $x y$-plane with the peakforce (PF) feedback enabled, while applying either a potential difference between the tip and the substrate (potentiostat) or forcing a fixed current through the tip (galvanostat). In all presented experiments, the peakforce frequency was set to $2 \mathrm{kHz}$, other AFM parameters used are listed in the supplementary file. Lift experiments where the tip is kept at a fixed distance above the substrate, as mentioned in the text, were done by initiating a Thermal Tune within the AFM software at a certain height.

Data treatment AFM images are treated by removing the polynomial background (first or second order) and removing image defects by aligning rows or removing scars using the Nanoscope Analysis and Gwyddion 56 software. The volume data in figure 2 is defined as the bearing volume in the deposited lines, with a threshold value for each pixel set to 2 times the sample roughness. The height data in figure $3 a$ is obtained by taking the average line profile of a written copper wire and fitting a Gaussian to obtain the height and full width half maximum as illustrated in Supplementary file SI-8. The volume in figure $3 \mathrm{~b}$ is then taken to be the area of this fit multiplied by the length of the path. Fitting was done using the Origin 2017 software.

Analytical model We have considered a mirror charge at a distance of $h_{\text {tip }}$ from the interface. $V_{\text {tip }}$ is defined as the potential at a radial distance of $25 \mathrm{~nm}$ and we have used the dielectric constant of water (80). We consider the substrate to be grounded with respect to the solution. This is a fair approximation given the much larger current density flowing through the tip.

\section{Acknowledgements}

This work is part of the research program at the Netherlands Organisation for Scientific Research (NWO). Authors thank D. Ursem and the design and precision manufacturing departments at Amolf for their support. We also thank M. Valenti, I. Kolpakov and A. Polman for fruitful discussions.

\section{Author information}

\section{Contributions}

M.A. prepared the samples and performed the experiments. E.A.L. and M.A. designed the study, analysed data, interpreted the results, developed the analytical modelling and wrote the paper.

\section{Competing interests}

The authors declare no competing financial interests.

\section{Corresponding author}

Correspondence to Esther Alarcon-Llado

\section{Supplementary information}

Supplementary Figures 1-?. 


\section{References}

1.Oja, S. M., Fan, Y., Armstrong, C. M., Defnet, P. \& Zhang, B. Nanoscale Electrochemistry Revisited. Analytical Chemistry 88, 414-430 (2015).

2.Staikov, G. Nanoscale electrodeposition of low-dimensional metal phases and clusters. Nanoscale 8, 13880-13892 (2016).

3.Hirt, L., Reiser, A., Spolenak, R. \& Zambelli, T. Additive Manufacturing of Metal Structures at the Micrometer Scale. Advanced Materials 29, 1604211 (2017).

4.Lam, B., Zhou, W., Kelley, S. O. \& Sargent, E. H. Programmable definition of nanogap electronic devices using self-inhibited reagent depletion. Nature Communications 6, (2015).

5.Schaedler, T. A. et al.. Ultralight Metallic Microlattices. Science 334, 962-965 (2011).

6.Gansel, J. K. et al.. Gold Helix Photonic Metamaterial as Broadband Circular Polarizer. Science 325, 1513-1515 (2009).

7.Reiser, A. et al.. Multi-metal electrohydrodynamic redox 3D printing at the submicron scale. Nature Communications 10, (2019).

8.Hu, J. \& Yu, M.-F. Meniscus-Confined Three-Dimensional Electrodeposition for Direct Writing of Wire Bonds. Science 329, 313-316 (2010).

9. Hirt, L. et al.. Template-Free 3D Microprinting of Metals Using a Force-Controlled Nanopipette for Layerby-Layer Electrodeposition. Advanced Materials 28, 2311-2315 (2016).

10.Momotenko, D., Page, A., Adobes-Vidal, M. \& Unwin, P. R. Write-Read 3D Patterning with a DualChannel Nanopipette. ACS Nano 10, 8871-8878 (2016).

11.Ghatkesar, M., Garza, H., Heuck, F. \& Staufer, U. Scanning Probe Microscope-Based Fluid Dispensing. Micromachines 5, 954-1001 (2014).

12.Maynor, B. W., Li, Y. \& Liu, J. Au "Ink" for AFM "Dip-Pen" Nanolithography. Langmuir 17, 2575-2578 (2001).

13.Je, J. H., Kim, J.-M. \& Jaworski, J. Progression in the Fountain Pen Approach: From 2D Writing to 3D Free-Form Micro/Nanofabrication. Small 13, 1600137 (2016).

14.Wang, F., Xiao, H. \& He, H. Effects of applied potential and the initial gap between electrodes on localized electrochemical deposition of micrometer copper columns. Scientific Reports 6, (2016).

15.LaGraff, J. R. \& Gewirth, A. A. Enhanced Electrochemical Deposition with an Atomic Force Microscope. The Journal of Physical Chemistry 98, 11246-11250 (1994).

16.Obermair, C., Wagner, A. \& Schimmel, T. The atomic force microscope as a mechano-electrochemical pen. Beilstein Journal of Nanotechnology 2, 659-664 (2011).

17.Vasko, S. E. et al.. Serial and Parallel Si Ge, and SiGe Direct-Write with Scanning Probes and Conducting Stamps. Nano Letters 11, 2386-2389 (2011).

18.Garcia, R., Knoll, A. W. \& Riedo, E. Advanced scanning probe lithography. Nature Nanotechnology 9, 577-587 (2014). 
19.Schindler, W., Hofmann, D. \& Kirschner, J. Localized Electrodeposition Using a Scanning Tunneling Microscope Tip as a Nanoelectrode. Journal of The Electrochemical Society 148, C124 (2001).

20.García, S. G., Salinas, D. R., Mayer, C. E., Lorenz, W. J. \& Staikov, G. STM tip-induced local electrochemical dissolution of silver. Electrochimica Acta 48, 1279-1285 (2003).

21.Wittstock, G., Burchardt, M., Pust, S. E., Shen, Y. \& Zhao, C. Scanning Electrochemical Microscopy for Direct Imaging of Reaction Rates. 1584-1617 (2007). doi:10.1002/anie.200602750

22.Sarkar, S., Lai, S. \& Lemay, S. Unconventional Electrochemistry in Micro-/Nanofluidic Systems. Micromachines 7, 81 (2016).

23. Liu, M. et al.. Enhanced electrocatalytic $\mathrm{CO} 2$ reduction via field-induced reagent concentration. Nature 537, 382-386 (2016).

24.Lee, S. S., Fenter, P., Nagy, K. L. \& Sturchio, N. C. Real-time observation of cation exchange kinetics and dynamics at the muscovite-water interface. Nature Communications 8, 15826 (2017).

25. Hugelmann, M. \& Schindler, W. Tunnel barrier height oscillations at the solid/liquid interface. Surface Science 541, L643-L648 (2003).

26.Collins, L., Kilpatrick, J. I., Kalinin, S. V. \& Rodriguez, B. J. Towards nanoscale electrical measurements in liquid by advanced KPFM techniques: a review. Reports on Progress in Physics 81, 086101 (2018).

27. Hiesgen, R., Eberhardt, D. \& Meissner, D. Direct investigation of the electrochemical double layer using the STM. Surface Science 597, 80-92 (2005).

28. Harniman, R. L. et al.. Real-time tracking of metal nucleation via local perturbation of hydration layers. Nature Communications 8, (2017).

29. Martin-Jimenez, D., Chacon, E., Tarazona, P. \& Garcia, R. Atomically resolved three-dimensional structures of electrolyte aqueous solutions near a solid surface. Nature Communications 7, (2016).

30.Fukuma, T., Kobayashi, K., Matsushige, K. \& Yamada, H. True atomic resolution in liquid by frequencymodulation atomic force microscopy. Applied Physics Letters 87, 034101 (2005).

31.Umeda, K., Kobayashi, K., Minato, T. \& Yamada, H. Atomic-Level Viscosity Distribution in the Hydration Layer. Physical Review Letters 122, (2019).

32.Voïtchovsky, K., Kuna, J. J., Contera, S. A., Tosatti, E. \& Stellacci, F. Direct mapping of the solid-liquid adhesion energy with subnanometre resolution. Nature Nanotechnology 5, 401-405 (2010).

33.Siretanu, I. et al.. Direct observation of ionic structure at solid-liquid interfaces: a deep look into the Stern Layer.. Scientific reports 4, 4956 (2014).

34. Hirata, K. et al.. Visualizing charges accumulated in an electric double layer by three-dimensional open-loop electric potential microscopy. Nanoscale 10, 14736-14746 (2018).

35.Perry, D., Botros, R. A., Momotenko, D., Kinnear, S. L. \& Unwin, P. R. Simultaneous Nanoscale Surface Charge and Topographical Mapping. ACS Nano 9, 7266-7276 (2015).

36.Xie, Z.-X. \& Kolb, D. M. Spatially confined copper dissolution by an STM tip: a new type of electrochemical reaction?. Journal of Electroanalytical Chemistry 481, 177-182 (2000). 
37.García, S. STM tip-induced local electrochemical dissolution of silver. Electrochimica Acta 48, 1279-1285 (2003).

38.Schuster, R., Kirchner, V., Allongue, P. \& Ertl, G. Electrochemical micromachining. Science 289, 98-101 (2000).

39. Collins, L. et al.. Probing charge screening dynamics and electrochemical processes at the solid-liquid interface with electrochemical force microscopy. Nature Communications 5, (2014).

40.Schuster, R., Kirchner, V., Xia, X. H., Bittner, A. M. \& Ertl, G. Nanoscale Electrochemistry. Physical Review Letters 80, 5599-5602 (1998).

41.Widmer, R. \& Siegenthaler, H. Nanostructuring experiments in the system $\mathrm{Ag}(111) / \mathrm{Pb} 2+$. Electrochemistry Communications 7, 421-426 (2005).

42.Potzschke R. T., Staikov, G., Lorenz, W. J. \& Wiesbeck, W. Electrochemical Nanostructuring of n-Si(111) Single-Crystal Faces. Journal of The Electrochemical Society 146, 141 (1999).

43. Huang, Z. et al.. PeakForce Scanning Electrochemical Microscopy with Nanoelectrode Probes. Microscopy Today 24, 18-25 (2016).

44.Kolb, D. M. \& Simeone, F. C. Electrochemical nanostructuring with an STM: A status report. Electrochimica Acta 50, 2989-2996 (2005).

45.Seol, S.-K., Pyun, A.-R., Hwu, Y., Margaritondo, G. \& Je, J.-H. Localized Electrochemical Deposition of Copper Monitored Using Real-Time X-ray Microradiography. Advanced Functional Materials 15, 934-937 (2005).

46. Madden, J. D. \& Hunter, I. W. Three-dimensional microfabrication by localized electrochemical deposition. Journal of Microelectromechanical Systems 5, 24-32 (1996).

47.Said, R. A. Microfabrication by localized electrochemical deposition: experimental investigation and theoretical modelling. Nanotechnology 14, 523-531 (2003).

48.Schuster, R. Electrochemical Micromachining. Science 289, 98-101 (2000).

49.Liu, Y., Gokcen, D., Bertocci, U. \& Moffat, T. P. Self-Terminating Growth of Platinum Films by Electrochemical Deposition. Science 338, 1327-1330 (2012).

50.Daryadel, S. et al.. Localized Pulsed Electrodeposition Process for Three-Dimensional Printing of Nanotwinned Metallic Nanostructures. Nano Letters 18, 208-214 (2017).

51.Quickenden, T. I. Toward a Reliable Value for the Diffusion Coefficient of Cupric Ion in Aqueous Solution. Journal of The Electrochemical Society 143, 1248 (1996).

52.Richard Compton, C. B. Understanding Voltammetry. in Understanding Voltammetry (IMPERIAL COLLEGE PRESS, 2011). doi:10.1142/9781848167322 0004

53.Bazant, M. Z., Thornton, K. \& Ajdari, A. Diffuse-charge dynamics in electrochemical systems. Physical Review E 70, (2004).

54.Yan, J. W. et al.. Formation and STM tip-induced reduction of ultra thin $\mathrm{SnO}$ film on $\mathrm{Au}(111)$. Chemical Physics Letters 373, 575-579 (2003). 
55.Sader, J. E. et al.. A virtual instrument to standardise the calibration of atomic force microscope cantilevers. Review of Scientific Instruments 87, 093711 (2016).

56.Nečas, D. \& Klapetek, P. Gwyddion: an open-source software for SPM data analysis. Open Physics 10, (2012). 


\title{
Supplementary File - Dynamic perturbation of the electrical double layer with an electrochemical AFM for confined metal electrodeposition
}

\author{
Mark Aarts and Esther Alarcon-Llado
}

SI-1. Elemental characterization of a larger deposit using SEM-EDX
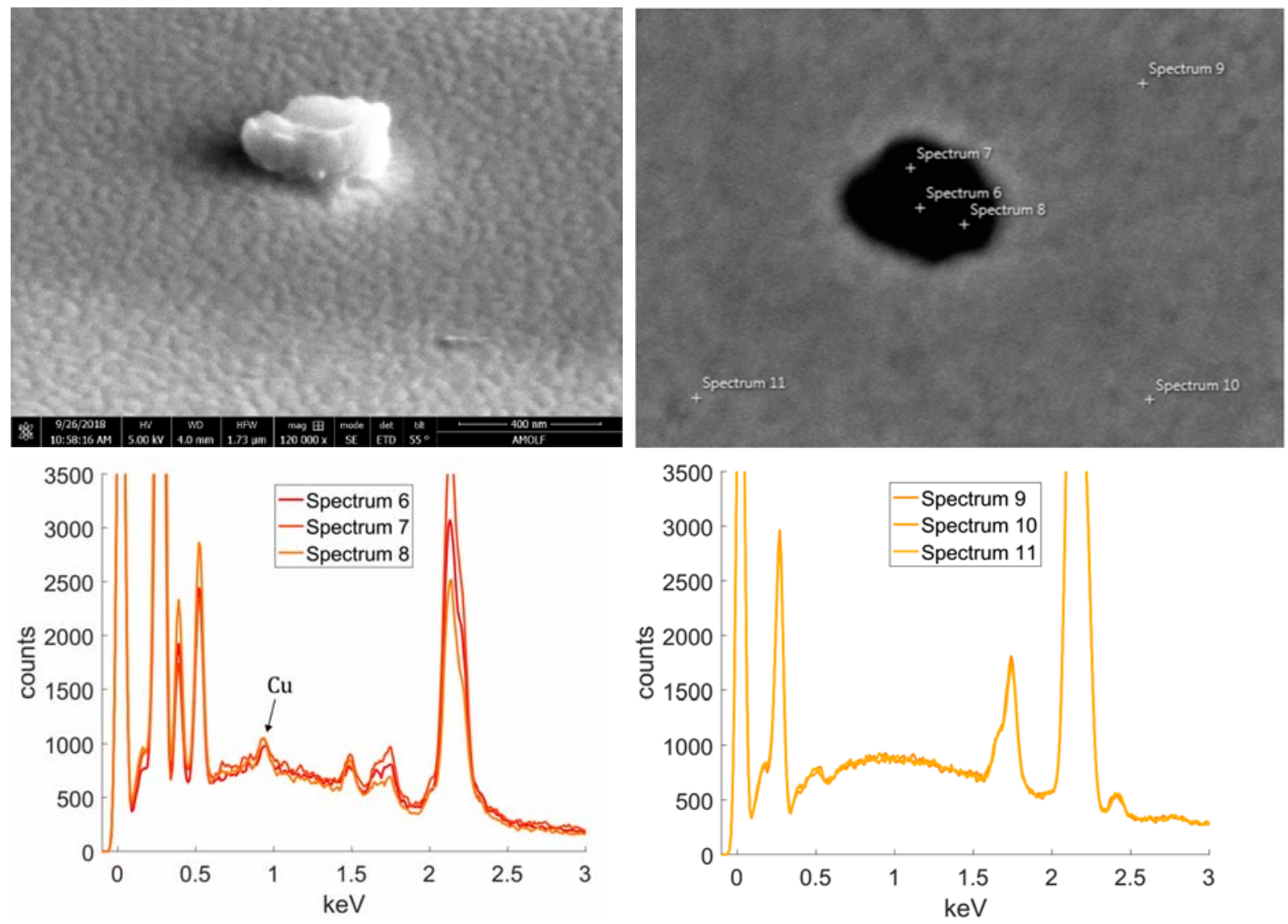

Supplementary Figure 1. Elemental characterization of a larger deposit using SEM-EDX

A larger structure ( $500 \mathrm{~nm}$ radius and $\sim 300 \mathrm{~nm}$ height as obtained from ex-situ AFM) was deposited with the tip stationary for an hour with a potential difference of $2 \mathrm{~V}$ between tip and substrate a) Secondary electron SEM image of the deposit (55 degrees tilt). B) Backscatter electron image at normal incidence, displaying elemental contrast between the deposit and the substrate. $C, D)$ EDX spectra of the points indicated on the deposit (C) and on the sides $(D)$, as indicated in (B). A clear peak associated with copper is observed at $\sim 0.94 \mathrm{keV}$, which is absent in the reference spectra. Peaks in the reference are associated with the substrate containing gold (2.13 keV) and silicon (1.74 keV), and carbon peaks (0.02, $0.27 \mathrm{keV})$. Additional peaks in the deposit include oxygen (0.52 keV), nitrogen (0.39 keV), and aluminum (1.49 keV), the last 2 of which we attribute to scattering inside the SEM chamber. Spectra were obtained with an acceleration voltage of $5 \mathrm{keV}$ and a current of $800 \mathrm{pA}$. 
SI-2. Extended parameters space for deposition in $10 \mu \mathrm{M} \mathrm{CuSO}_{4}$ and $\mathrm{I}-\mathrm{V}$ characteristics as a function of ion concentration
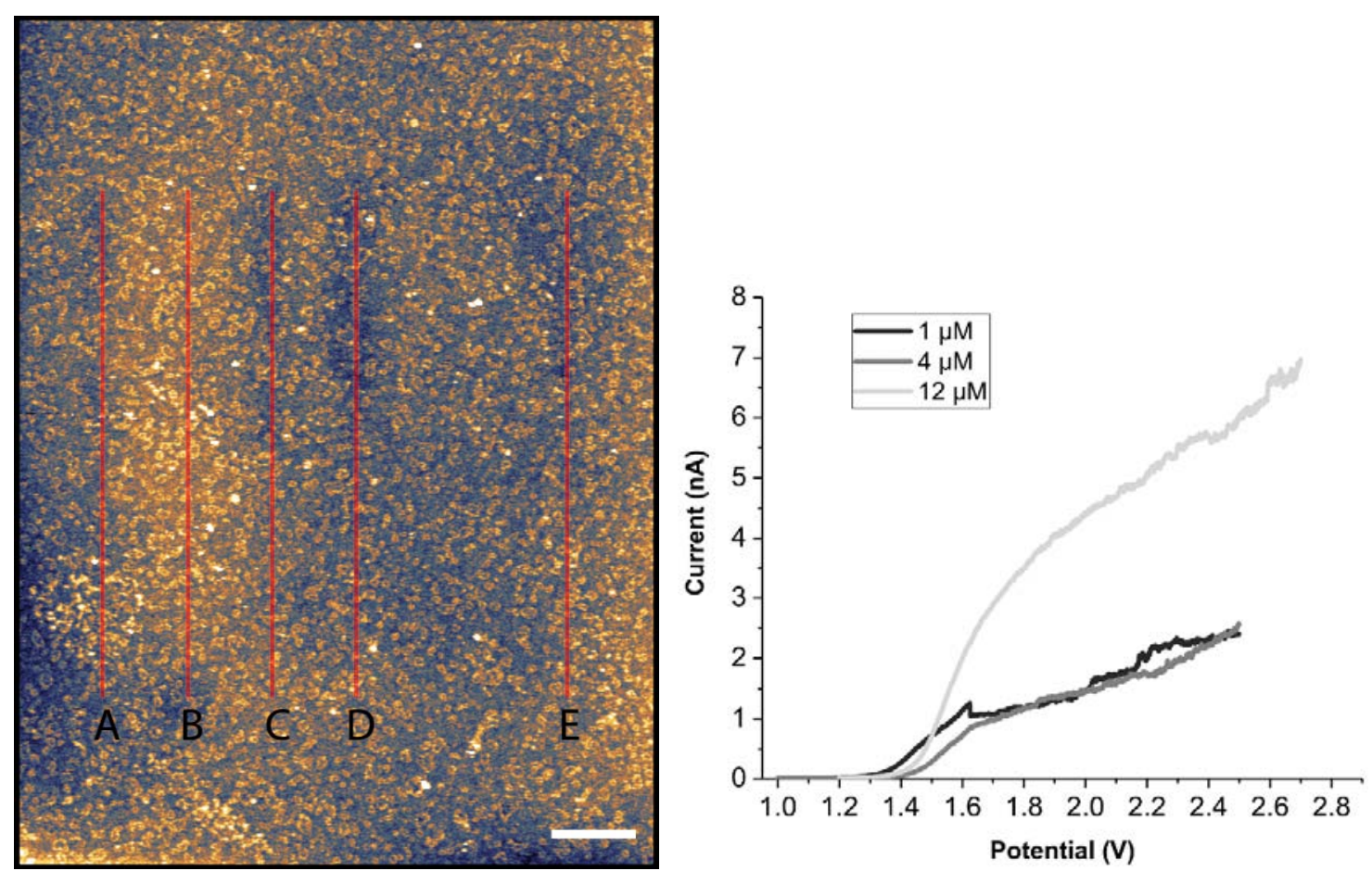

Supplementary Figure 2. Extended parameters space for deposition in $10 \mu \mathrm{M} \mathrm{CuSO}_{4}$

Left panel) Larger scan area for the highest concentration (12 $\mu \mathrm{M})$ of the image shown in the main text in figure $1 \mathrm{c}$. The tip is translated along the red lines (guide to the eye), while ramping the voltage at 1.25 or $2.5 \mathrm{mV} / \mathrm{s}$, and using different force setpoints for the feedback while moving. The translation speed is $10 \mathrm{~nm} / \mathrm{s}$ for line $A$ (main text), and $20 \mathrm{~nm} / \mathrm{s}$ for $B$ through $E$. The force is varied in terms of the photodiode voltage (Supplementary file 6.), $0.1,0.15,0.1$, $0.05,0.05 \mathrm{~V}$ for line A through $\mathrm{E}$, respectively. The voltage is ramped from $1.2->2.7,1.2->1.9,1.2->2.7,1.2->2.7$, $1->2.5 \mathrm{~V}$ in the same order. No deposition is observed for any of the parameters in the vicinity of our experiment at this concentration.

Right) The current measured at the nanoelectrode while walking the paths as shown in figure $1 d$ in the main text. The tip potential is swept from $1->2.5 \mathrm{~V}$ for the 1 , and $4 \mu \mathrm{MCuSO}_{4}(\mathrm{aq})$ solution, and from 1 -> $2.7 \mathrm{~V}$ for the $12 \mu \mathrm{MCuSO} \mathrm{C}_{4}$ (aq) solution. The path is walked over a period of 20 minutes with a sweep rate of $1.25 \mathrm{mV} / \mathrm{s}$ and a current sample interval of $1 \mathrm{mV}$. 


\section{SI-3. Contact time and center of oscillation with AFM parameters}
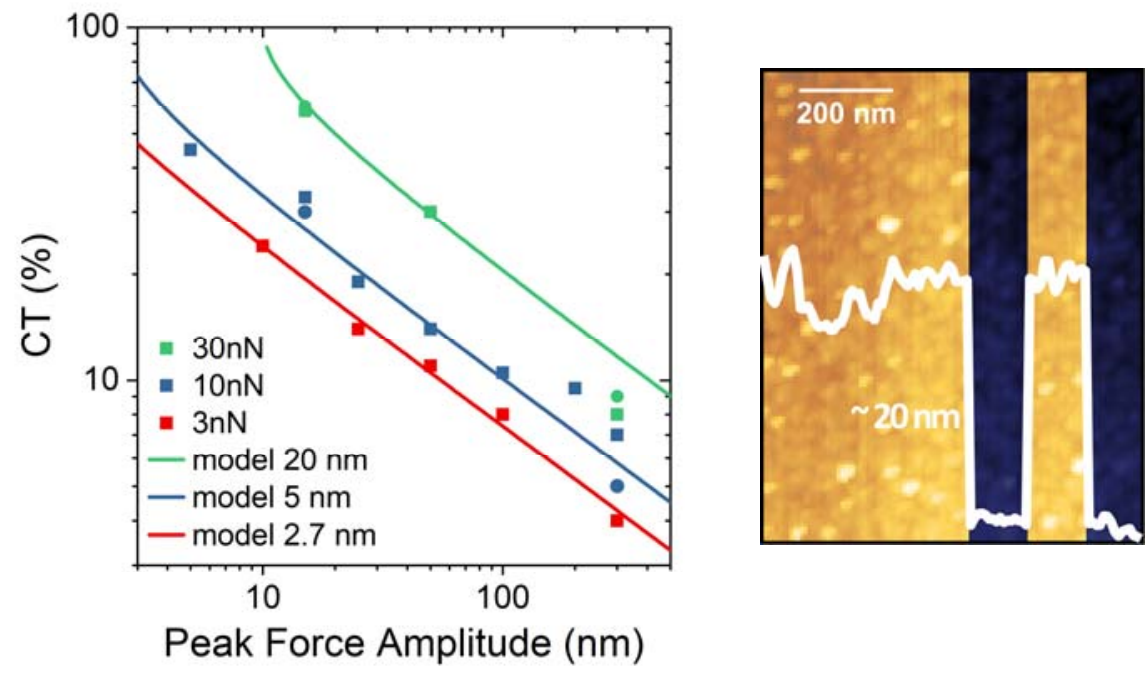

Supplementary Figure 3. Dependence of cantilever position on AFM parameters

The average gap size $<z>$, as referred to in the main text, is determined by integrating the tip position from the highest point up to the point of contact, divided by half the oscillation period.

$\frac{\int_{0}^{\frac{T-C T}{2}} P F A * \cos \left(\frac{f}{2 \pi} t\right) d t}{\frac{T}{2}}$, with CT the measured contact time, PFA the peakforce amplitude and using the frequency $f=2 \mathrm{kHz}$, and period $T=500 \mu \mathrm{s}$.

The figure illustrates the effect of peak force amplitude and peak force setpoint on the amount of time spent in contact with the sample. Left) Contact time read out from the Nanoscope software for the contact time as a function of peakforce amplitude for different force setpoints (dots, squares). The center of the sinusoidal oscillation is expected to shift by the force setpoint divided by the spring constant, assuming no indentation of the sample. This gives 2, 6.7, and $20 \mathrm{~nm}$ for a setpoint of 3, 10, and $30 \mathrm{nN}$ respectively, for the nominal spring constant of $1.5 \mathrm{~N} / \mathrm{m}$. The straight lines are a fit to the data calculated for a sinusoidal movement of the tip with the center of the oscillation given by the amplitude-x, yielding $x=2.7,5$, and $20 \mathrm{~nm}$ for the different forces, in good agreement with the expected shift.

Right) Raw AFM height sensor data when switching the peakforce setpoint from $3 n N->30 n N$ twice during the scan (slow scan axis in horizontal direction). The overlay shows the height profile, illustrating how the Z-piezo moves down (approximately $20 \mathrm{~nm}$ ) due to the force setpoint, determined by the cantilever spring constant and indentation of the sample. 


\section{SI-4. Width of deposited lines}

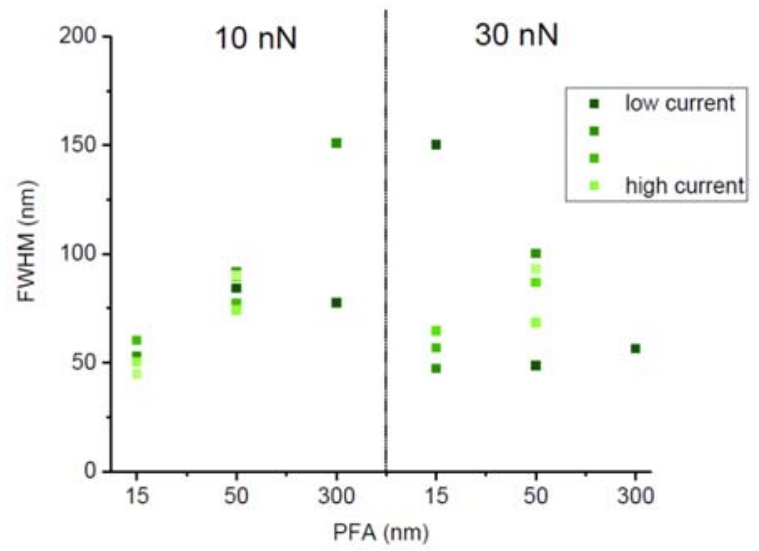

\section{Supplementary Figure 4. FWHM of deposited lines from Figure $3 b$ in the main text}

The full width half maximum of the lines used for figure $3 \mathrm{~b}$ in the main text. The individual datapoints for all currents are displayed, where the color ranges from dark to light green, for lower to higher currents, respectively (arbitrary scale). The amount of individual current datapoints varies for each set of oscillation parameters, as growth is not observed for all currents (see main text). While the width seems to increase slightly at the intermediate amplitude, no clear trends in the width are observed as a function of the applied current.

\section{SI-5. Current response of the nanoelectrode in consecutive approach curves}

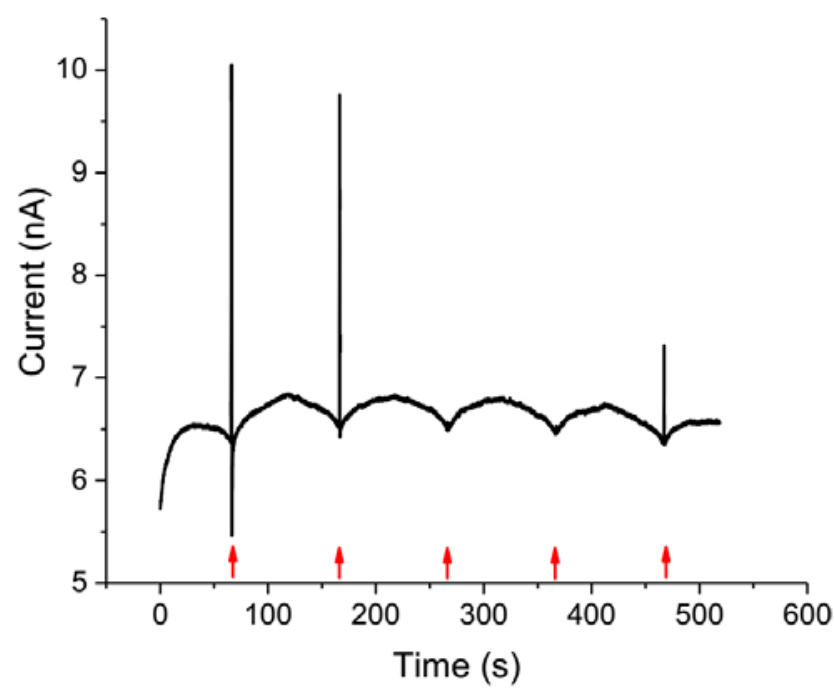

\section{Supplementary Figure 5. Current response of nanoelectrode during approach}

Current response at the AFM nanoelectrode when executing 5 consecutive approaches with a tip potential of $1.7 \mathrm{~V}$. The tip is approached and retracted over a distance of $1 \mu \mathrm{m}$ with a frequency of $0.01 \mathrm{~Hz}(20 \mathrm{~nm} / \mathrm{s})$. These approach curves show that the current decreases/increases as the tip approaches/retracts from the surface. At the point of contact (indicated by the red arrows), a short circuit current is observed. The current sampling rate is $20 \mathrm{~Hz}$. The approach is terminated at an applied force of $\sim 10 \mathrm{nN}$ (deflection trigger of $8 \mathrm{~nm}$ with a spring constant of $1.25 \mathrm{~N} / \mathrm{m}$ ) 
SI-6. Electrostatic interaction between biased AFM-tip and substrate in liquid
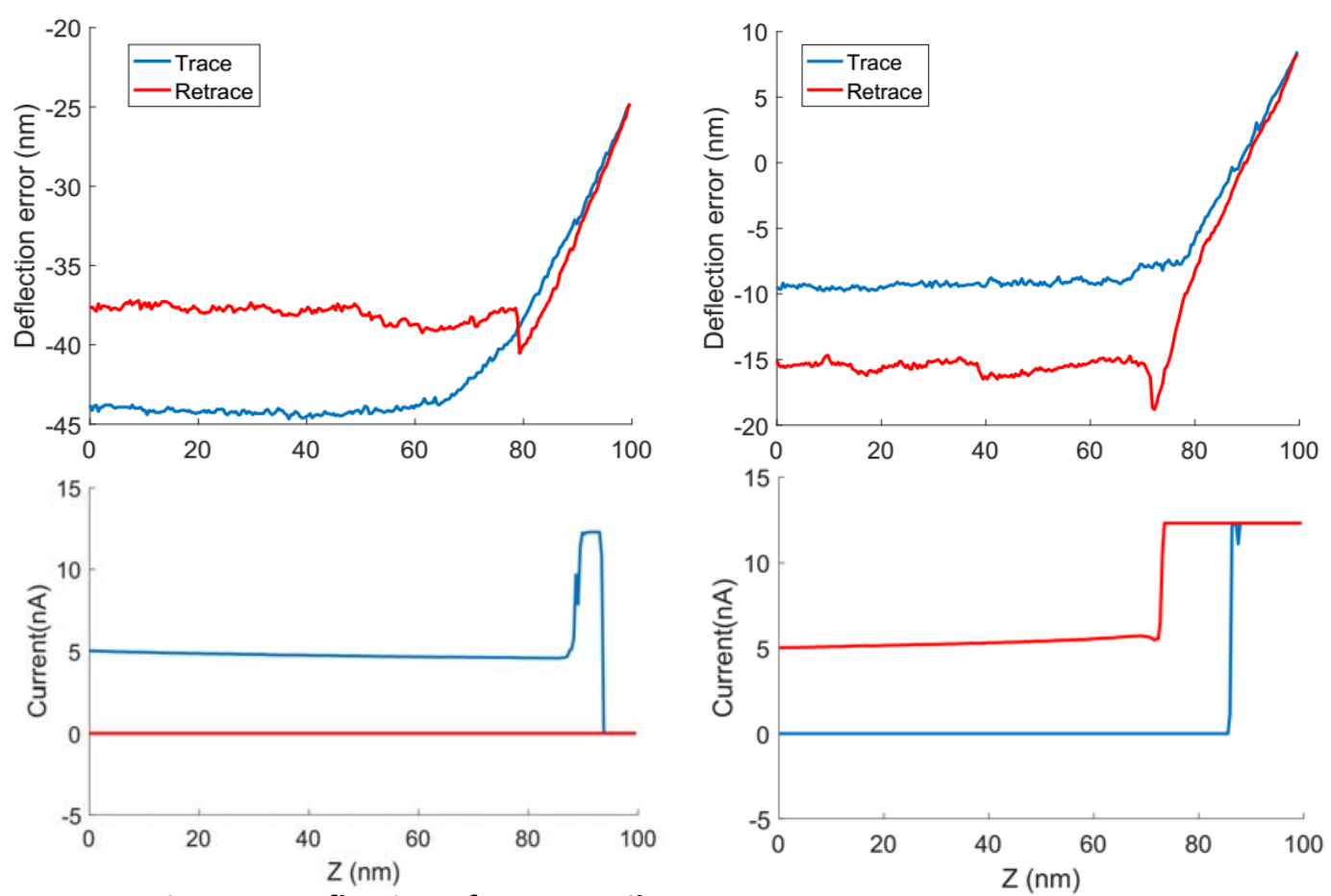

Supplementary Figure 6. Deflection of AFM cantilever in liquid when applying a potential

Single approach curve of the AFM tip where the tip-substrate potential was manually turned off for the retrace during the contact( left panels), and turned on for the retrace during the contact (right panels), as seen in the current channel (bottom panels) that is measured simultaneously by the AFM software.

The applied potential is $2.1 \mathrm{~V}$. In both cases a clear attractive force is seen in the deflection of the cantilever (top panels) when a potential is applied (as evidenced by the flow of current in the bottom panels). The difference in deflection of $\sim 5 \mathrm{~nm}$ corresponds to $\sim 4 \mathrm{nN}$ for this spring constant.

The ramp size is $100 \mathrm{~nm}$, at $0.01 \mathrm{~Hz}(2 \mathrm{~nm} / \mathrm{s})$, the force-trigger for the approach curve was set to 15 $\mathrm{nN}$ (deflection trigger of $17 \mathrm{~nm}$ with a spring constant of $1.15 \mathrm{~N} / \mathrm{m}$ ).

\section{SI-7. AFM parameters and cantilever resonance characteristics}

Table listing the resonance characteristics and the spring constants of all cantilevers used.

\begin{tabular}{|l|l|l|l|}
\hline Data used in & Peakforce Amplitude $(\mathrm{nm})$ & Peakforce setpoint $(\mathrm{nN})$ & Translation speed $(\mathrm{nm} / \mathrm{s})$ \\
\hline Fig 1b & 50 & 30 & 3 \\
\hline Fig 1c & 50 & 30 & $30+3$ (consecutive) \\
\hline Fig 1d & 50 & $6.3+$ & 10 \\
\hline Fig 2b & 50 & 30 & 10 \\
\hline Fib 2c & 50 & 30 & $3,10,30,100$ \\
\hline Fig 3b & $15,50,300$ & 10,30 & 10 \\
\hline Fig 3c & $10,25,50,100,300$ & 3 & 10 \\
\hline S.1. & 50 & 30 & - \\
\hline
\end{tabular}




\begin{tabular}{|l|l|l|l|}
\hline S.2. & 50 & $2.1,3.2,6.3,9.5 \dagger$ & 10,20 \\
\hline S.4. & $15,50,300$ & 10,30 & 10 \\
\hline
\end{tabular}

† The force was set in terms of the photodiode voltage. With a used deflection sensitivity of $35 \mathrm{~nm} / \mathrm{v}$ and a spring constant that was later determined to be 1.8 (see next table for the resonant behavior), this yields a force of $6.3 \mathrm{nN}$ for the used setpoint voltage of $0.1 \mathrm{~V}$.

\begin{tabular}{|l|l|l|l|}
\hline Data used in & Frequency in air $(\mathrm{kHz})$ & Q-factor in air & Used k (N/m) \\
\hline Fig 1b & $65.7^{\dagger}$ & $208^{\dagger}$ & 1.68 \\
\hline Fig 1c & $32.6^{\ddagger}$ & - & 1.80 \\
\hline Fig 1d & $63.4^{\dagger}$ & $214^{\dagger}$ & - \\
\hline Fig 2 & $65.7^{\dagger}$ & $208^{\dagger}$ & 1.68 \\
\hline Fig 3b & 58.3 & 195 & 1.41 \\
\hline Fig 3c & $63.4^{\dagger}$ & $210^{\dagger}$ & 1.59 \\
\hline S.1. & $51.3^{\dagger}$ & $116^{\dagger}$ & 0.88 \\
\hline S.2. & $63.4^{\dagger}$ & $214^{\dagger}$ & - \\
\hline S.4. & 58.3 & 195 & 1.41 \\
\hline S.5. & 58.3 & 200 & 1.25 \\
\hline S.6. & $52.1^{\dagger}$ & $105^{\dagger}$ & 1.15 \\
\hline
\end{tabular}

+ Frequency and Q-factor were obtained in air for that tip on a different day than the day of the measurement, the reported spring constant is the value used on the day of the measurement in the electrolyte.

₹ Frequency was obtained with the tip immersed in the liquid 
SI-8. Line profile fitting and analysis
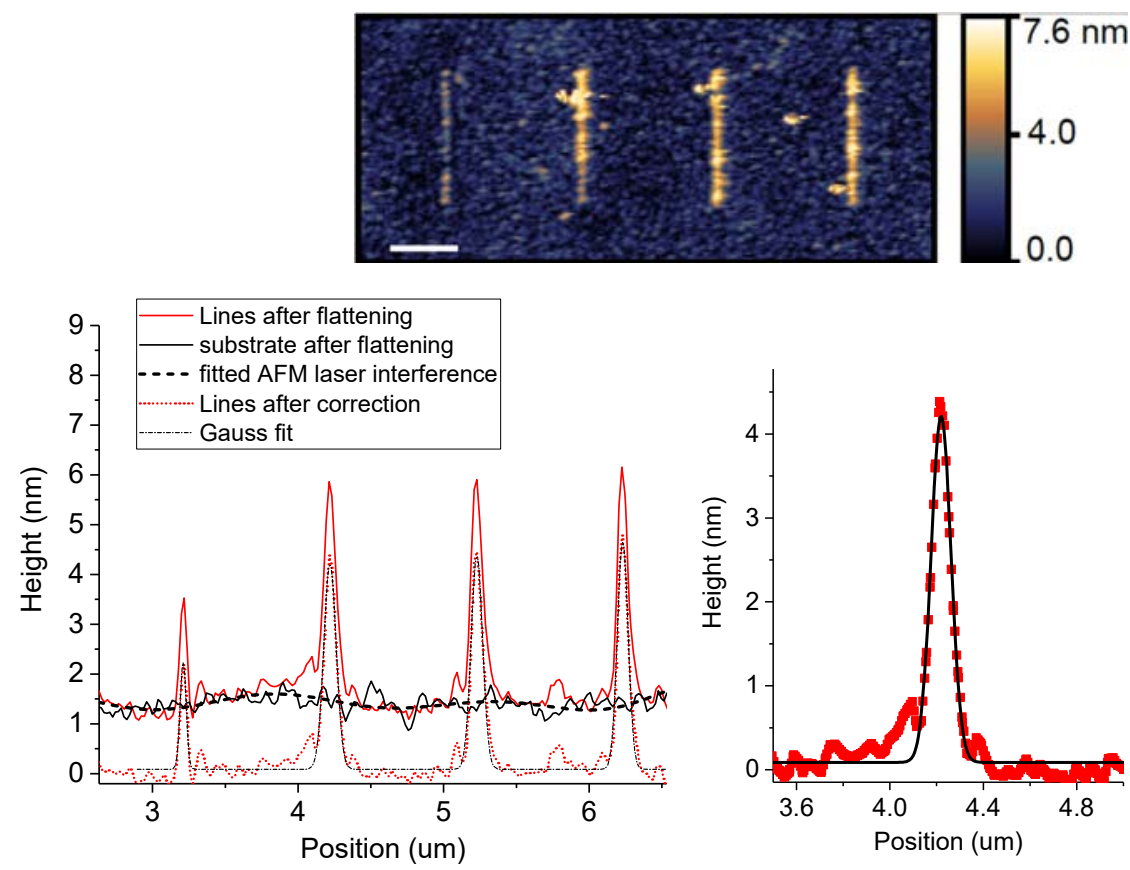

Supplementary Figure 8. Illustration of the line profiles and fitting used for analysis

Top: Representative AFM images used in the main text (fig $3 \mathrm{~b}, 30 \mathrm{nN}$ peakforce $50 \mathrm{~nm}$ peakforce amplitude, for different currents) scale bar $500 \mathrm{~nm}$. Bottom left: Averaged profile of the lines along the vertical direction (solid red curve). The solid black curve is the average profile of the substrate outside the lines used to account for the laser interference (dashed black curve). The dotted red line is the background corrected average line profile. Thin dash-dotted line is the Gaussian fit to the four lines. Bottom right: zoom-in of the profile and fit for the line second from the left. The Gauss function in Origin 2017 was fitted, $y=y_{0}+\frac{A}{w \sqrt{\left(\frac{\pi}{2}\right)}}+e^{-\frac{2\left(x-x_{c}\right)^{2}}{w^{\wedge} 2}}$, with $y_{0}$ being the offset, $x_{c}$ the center position, and w equal to $\sqrt{\ln (4)^{*}}$ the full width at half maximum. 
